\title{
Single and Multiple Electron Dynamics in the Strong Field Limit
}

\author{
B. Sheehy \\ B. Walker \\ R. Lafon \\ M. Widmer \\ A. Gambhir \\ L.F. DiMauro \\ P. Agostini \\ K.C. Kulander \\ This paper was prepared for submittal to the \\ 7th International Conference on Multiphoton Processes \\ Garmisch-Partenkirschen, Germany \\ September 29-October 4, 1996
}

October 1996

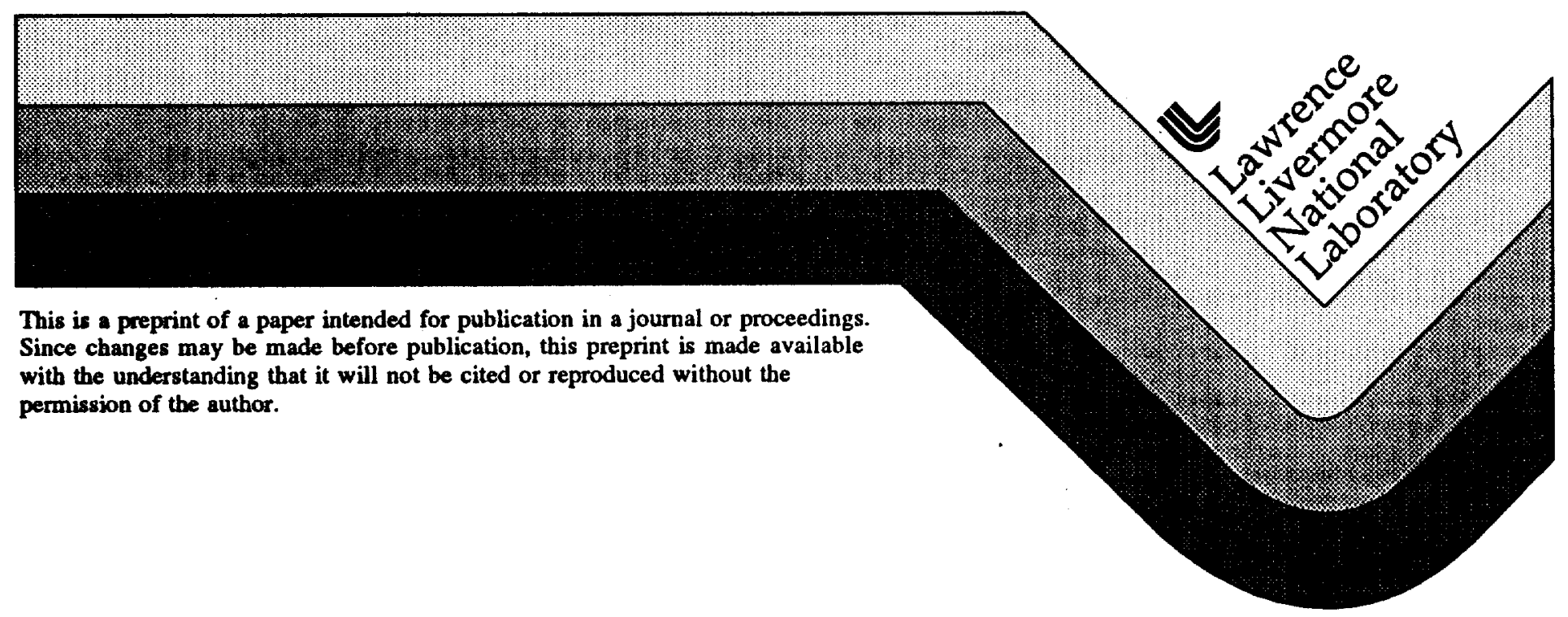


This document was prepared an account of work sponsored by an agency of the United States Government. Neither the United States Government nor the University of California nor any of their employees, makes any warranty, express or implied, or asumes any legal liability or responsibility for the accuracy, completeness, of unefulnese of any information, apparatus, product, or process disclosed, or represents that its wee would not Infringe privately owned rights. Reference herein to any specific commercial product, process, or service by trade name, trademerk, manufacturer, or otherwise, does not necesenrily constitute or imply its endonsement, recommendation, or favoring by the United States Government or the Univeratty of California. The views and opinions of authose expremed herein do not neceserrily state or reflect those of the United States Government or the University of Callfornin, and shall not be used for advertiaing ar product endorsement purposece. 


\title{
Single and multiple electron dynamics in the strong field limit
}

\author{
B Sheehyt, B Walkert, R Lafont, M Widmert, A Gambhirt, L F \\ DiMaurot, P Agostinif and K C Kulander \\ $\dagger$ Brookhaven National Laboratory, Department of Chemistry, Upton, NY \\ 11973 , US \\ $\ddagger$ Service des Photons, Atomes et Molécules, Centre d'Etudes de Saclay, \\ 91191 Gif Sur Yvette, France \\ h TAMP Group, Lawrence Livermore National Laboratory, Livermore, CA \\ 94551, US
}

\begin{abstract}
High precision photoelectron energy and angular distributions in helium and neon atoms for a broad intensity range reflect the change in the continuum dynamics that occurs as the ionization process evolves into the pure tunneling regime. Elastic rescattering of the laser-driven free electron from its parent ion core leaves a distinct signature on the spectra, providing a direct quantitative test of the various theories of strong field multiphoton ionization. We show that it takes a relatively complete semi-classical rescattering model to accurately reproduce the observed photoelectron distributions. However, the calculated inelastic rescattering rate fails to reproduce the measured nonsequential double ionization yields.
\end{abstract}

\section{Introduction}

Strong field photoelectron spectra have attracted considerable attention over the past decade and a half, but a comprehensive understanding of the underlying dynamics which produce these spectra and related phenomena has solidified with the development of high repetition-rate, short pulse lasers which can span the entire intensity range of importance [1]. In 1957 Keldysh [2] showed that at infrared and visible wavelengths the dynamics of strong field atomic ionization undergoes a change in character as the laser intensity increases. In weak fields electrons are promoted into the continuum by the simultaneous absorption of enough photons to increase their energy above the ionization potential. This is called multiphoton ionization (MPI). However, as the laser intensity increases, a completely separate mode of escape becomes possible. At large distances from the nucleus the electrostatic attraction of the ion core can be overwhelmed by the laser's 
instantaneous electric field, producing a barrier through which a valence electron can tunnel. In this regime a quasi-static tunneling picture becomes appropriate: the laser field varies so slowly compared to the response time of the electron that the ionization rate becomes simply the cycle-average of the instantaneous dc-tunneling rate. Tunneling becomes important when the ratio of the frequency of the applied field to the tunneling rate becomes smaller than unity. This ratio, known as the Keldysh or adiabaticity parameter, $\gamma$, is given by $\left[I_{p} /\left(2 U_{p}\right)\right]^{1 / 2}$, where $I_{p}$ is the binding energy of the electron and $U_{p}=I / 4 \omega^{2}$ is the ponderomotive energy in atomic units of a free electron in the laser field of frequency $\omega$ and intensity $I$.

The majority of experimental studies on neutral atoms exposed to intense, shortpulse laser fields have been carried out in the MPI regime $(\gamma \geq 1)$. A few experiments $[3,4]$ have extended into the tunneling regime, but these measurements have been limited to observations of total ionization rates or electron energy distributions over a small dynamic range. In this paper, we report upon the first systematic experimental investigation in the strong field tunneling limit. Our study, by virtue of the enhanced dynamic range accessible with kilohertz laser technology, follows the change in the ion yield curves, electron spectrum and angular distributions as the ionization evolves from predominantly MPI to pure tunneling. The study examines both helium and neon atoms which have been established to tunnel ionize near the saturation intensity[5]. We find that the electron distributions in the tunneling regime are very different from any previous reports obtained in the MPI or mixed regimes $[6,7,8]$. We achieve a quantitative description of the electron spectra using a rescattering picture[8, 9] which mimics the time evolution of a tunnel-ionized continuum wave packet in the combined fields of the laser and ion core. However, the same model when applied to the $\mathrm{e}-2 \mathrm{e}$ inelastic rescattering process fails, both quantitatively and qualitatively, to describe the measured nonsequential production of doubly charged helium and neon ions. Since rescattering events are known to be important in other short-pulse, strong-field emission phenomena (e.g., harmonic generation) this investigation better defines the underlying dynamics of these processes.

\section{Method}

\subsection{Experimental}

In the experiments presented here a $120 \mathrm{fs}, 1 \mathrm{kHz}$ repetition rate, titanium sapphire laser operating at $0.78 \mu \mathrm{m}$ was focused by $\mathrm{f} / 4$ optics into an ultrahigh vacuum chamber. Under these conditions, the system is capable of producing a maximum intensity of $20 \mathrm{PW} / \mathrm{cm}^{2}$ with typical pulse-to-pulse fluctuations $<1.7 \%$ for $10^{6}$ laser shots. The studies at 0.39 $\mu m$ were performed by frequency doubling the output in a $0.7 \mathrm{~mm}$ thick BBO crystal. At this wavelength a maximum intensity of $2.5 \mathrm{PW} / \mathrm{cm}^{2}$ is obtained using $\mathrm{f} / 4$ optics. The laser polarization is $>99 \%$ over the entire intensity range. The laser intensity is calibrated by measuring the short pulse resonant photoelectron spectrum of xenon as a function of pulse energy and concurrently recording the xenon and helium or neon total ion yields. The calibration is corroborated by a spot size-measurement and accurate to approximately $25 \%$. The helium and neon samples were high purity $99.999 \%$ gases which were further scrubbed to $<0.1$ ppm for $\mathrm{O}_{2}, \mathrm{H}_{2}, \mathrm{H}_{2} \mathrm{O}, \mathrm{CO}_{2}$ and hydrocarbon impurities. A $30 \mathrm{~cm}$ long time-of-flight (TOF) photoelectron (PE) spectrometer provides energy and angular resolution of $0.05 \mathrm{eV}$ and $65 \mathrm{mrad}$, respectively. The spectrometer's energy 
calibration was obtained by recording the long pulse, high order ( $>40$ photon absorption) above-threshold ionization spectrum of xenon. A $30 \mathrm{~cm}$ TOF mass spectrometer provides sufficient resolution $(m / \delta m>300)$ to easily separate $\mathrm{H}_{2}^{+}$and $\mathrm{He}^{2+}$ mass peaks. The $e / m$ detection sensitivity for the singly and doubly charged ions is determined from the yields at the saturation intensity. Data collection used $1 \mathrm{~ns}$ binning of discriminated electron and ion events operating at low event probability ( $\leq 0.25 /$ shot), ensuring space charge free conditions. The current PE spectra were recorded between 0.5 and 1.5 times the saturation intensity $\left(I_{s a t}^{H e}=0.8 \mathrm{PW} / \mathrm{cm}^{2}\right.$ and $\left.I_{s a t}^{N e}=0.6 \mathrm{PW} / \mathrm{cm}^{2}\right)$ for neutral helium and neon, respectively. Walker et al [5] showed that contributions due to sequential ionization of $\mathrm{He}^{+}$remain insignificant up to an intensity of $\sim 4 \mathrm{PW} / \mathrm{cm}^{2}$, while the current study shows a similar behavior for neon.

\subsection{Quasiclassical model in the tunneling limit}

The demarcation between the ionization pathways mentioned above becomes apparent when the wave packets promoted from the bound state into the continuum in these two limiting cases are considered. In the MPI regime, ionized population appears continuously, at all phases of the field. To conserve momentum, this transition must take place while the electron is close to the nucleus producing a wave packet, initially localized near the ion core. When the intensity increases to the point that $\gamma<1$, tunneling begins to dominate and the electron is released a significant distance from the nucleus. During a narrow time interval near the maximum in the oscillating electric field, an asymmetric, delocalized wave packet emerges at the outer edge of the suppressed Coulomb barrier. However the overall symmetry of the ionization process is maintained by a complementary wave packet being generated on the opposite side of the nucleus one-half cycle later. Clearly, the evolution of the continuum wave packets in these two limiting cases can be very different- which is reflected in our experiment.

The motion of the tunneling wave packet is controlled mostly by its interaction with the laser field since it rapidly moves beyond the range where the ion core potential is effective. Its evolution can be reasonably approximated using the classical equations of motion for a laser-driven electron. In a simple quasiclassical (SQC) model [3], the bound electron becomes free at a particular phase, $\omega t_{o}$, of the field, then undergoes oscillatory motion at the laser frequency, $\omega$. The electron-field interaction in the length gauge can be written as $e \mathcal{E} \hat{z} \sin \omega t$, where $\hat{z}$ is the direction of polarization and $\mathcal{E}=(8 \pi I / c)^{1 / 2}$ is the amplitude of the laser's electric field. Assuming that the electron is initially at rest after tunneling, the time dependent electron velocity is given in atomic units as $v(t)=(\mathcal{E} / \omega) \times\left(\cos \omega t-\cos \omega t_{0}\right)$, where the first term is the field-induced quiver motion and the second term the drift velocity which is established the instant the electron appears in the continuum. In a short pulse experiment, the detected photoelectron energy corresponds to this drift energy. Therefore in the absence of further interactions with the ion core (rescattering) the maximum drift energy an electron can have is $2 U_{p}$.

The rescattering picture $[8,9]$ goes beyond the SQC model by recognizing that although in the tunneling regime electrons released into the field are initially accelerated away from the ion core, roughly half the electrons will recross the plane of the nucleus when the field has changed sign. During this interval the electron wave packet propagates for at least half an optical cycle beyond the range of the ion core potential, spreading freely in the transverse directions. Classical analysis $[7,10]$ shows that photoelectrons with energies $>2 U_{v}$ are produced by trajectories which experience very large deflections 
(elastic backscattering, $\theta>\pi / 2$ ) when they rescatter from the ion core. The electron's scattering angle and final drift energy are closely correlated. The elastic differential cross section in the backward direction can be approximated by that for Rutherford scattering, $\left[\sigma(\theta) \sim\left(1 / E^{2}\right) \times c s c^{4}(\theta / 2)\right][11]$, which varies slowly over the critical angular range producing the flat energy distribution for these PEs. The hard collisions (small impact parameters) necessary for producing large changes in drift energy also results in broader angular distributions for the high energy electrons.

We can extend the SQC model to include the effect of the first rescattering on the tunneling wave packet. We divide the optical cycle into a large number of equal time intervals. In each interval a trajectory is launched at the outer turning point of the suppressed effective potential with zero velocity. It is propagated in the combined fields of the laser and the helium(neon) ion core until either it escapes or returns to cross the plane of the nucleus. Those which escape contribute to the spectrum below $2 U_{p}$ according to their drift velocities as in the SQC model. The returning trajectories are assumed to be guiding a freely spreading gaussian wave packet whose width is given by $\alpha(\tau)=$ $\left(\alpha(0)^{2}+[2 \tau / \alpha(0)]^{2}\right)^{1 / 2}$, where $\alpha(0)$ is the initial width and $\tau$ is the propagation time between initiation and return. Choosing $\alpha(0)=4.0 a_{0}$, gives a return width consistent with our numerical studies [5]. We calculate the differential elastic scattering cross section for this wave packet using [11]

$$
\sigma(\theta)=\left|\frac{1}{2 i k} \sum_{\ell=1}^{\ell_{\max }} a_{\ell}(2 \ell+1) e^{2 i\left(\eta_{\ell}+\delta_{\ell}\right)} P_{\ell}(\cos \theta)\right|^{2} .
$$

Here $\delta_{\ell}$ is the Coulomb phase shift (for a clarge of 1 ) and $\eta_{\ell}$ is the additional phase shift resulting from the short range part of the $\mathrm{He}^{+}\left(\mathrm{Ne}^{+}\right)$potential. These phase shifts are obtained from numerical integration of the scattering equations for electron- $\mathrm{He}^{+}\left(\mathrm{Ne}^{+}\right)$ over the necessary range of energies and angular momenta. The partial wave amplitudes, $a_{\ell}$ are determined from the distribution of impact parameters in the returning wave packet $\left(\ell=m v_{\text {ret }} b\right)$.

Equation (1) gives the field-free differential cross section. The laser field will distort this distribution. The transverse component of the outgoing velocity is conserved, but the velocity along the polarization direction has both a drift component and a quiver velocity which depend on the phase of the laser field at the return time. Drift velocities corresponding to $\mathrm{PE}$ energies as high as $10 U_{p}$ can be produced if the trajectory is scattered by $\sim 180^{\circ}$. The energy and angular distributions from the wave packet in each time interval are weighted by the instantaneous tunneling rate. In these calculations we have actually used a scaled dc-tumneling rate which, when cycle averaged, gives the ADK rate [12]. This accounts for the initial state not being purely hydrogenic. The total angle-resolved electron distribution for a given laser intensity is obtained by summing the contributions from all time intervals. The e-2e inelastic process, which leads to the production of double ionization, is calculated using a modified Lotz cross section [13] which accounts for both excitation and ionization. Spatial and temporal averaging is performed for comparison to the experimental measurements.

\section{Tunneling photoelectron distributions}

Figure 1 shows the experimental angle-resolved PE spectrum (ARPES) for helium at the saturation intensity. Evident is the preponderance of $\mathrm{PE}$ energies lying below $2 U_{v}$ which 


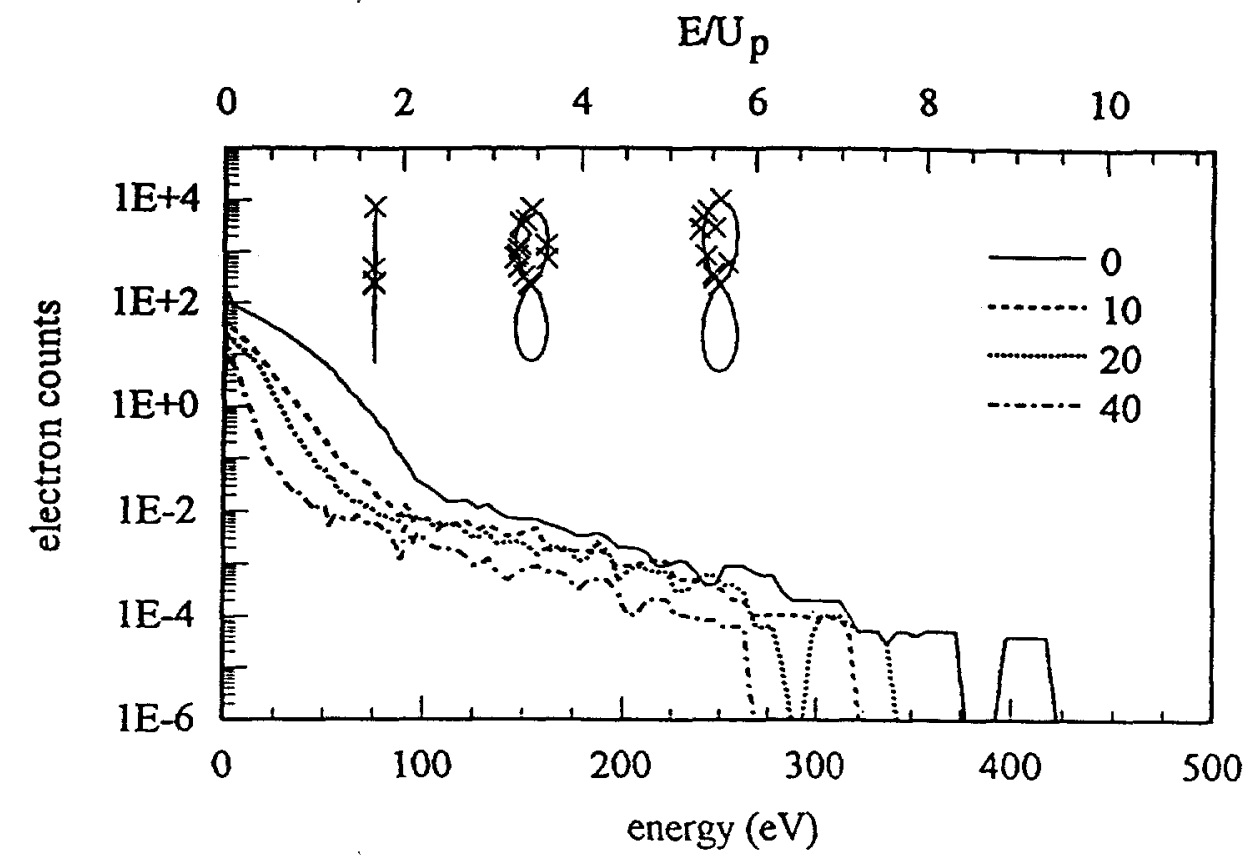

Figure 1. Measured helium ARPES for four different emission angles at the saturation intensity. The polar plots show the measured ADs (crosses) at the indicated energies and the solid lines are only to guide the reader. The laser polarization direction is vertical.

is consistent with the simple quasi-classical (SQC) model [3]. The angular distributions (AD) of these electrons are strongly aligned along the laser polarization direction (see polar inset), becoming narrower towards the $2 U_{p}$, limit. However, a striking change occurs in the $\mathrm{AD}$ of electrons with energies above $2 U_{p}$. Here the ADs are significantly broader with a weak narrowing as the energy increases towards $10 U_{p}$, indicative of rescattering. In fact, this ARPES seems to be a superposition of two components: a "normal" narrow distribution that falls off rapidly with increasing energy between 0 and $2 U_{p}$ and a much broader but weaker, almost flat energy distribution that extends out to high energies before abruptly truncating at $\delta-10 U_{p}$. This differs dramatically from all previous experimental reports [1]. For example, the $\mathrm{PE}$ distributions for inert gas atoms [6, 7] clearly show angle-dependent structures, as well as an abundance of electrons with energies $>2 U_{n}$. These differences reflect the pure tumneling nature of these experiments and provides a unique opportunity for quantitatively testing the rescattering picture.

Total PE distributions are shown in Fig. 2 for three different intensities in both absolute and scaled energy units. The distributions are constructed by integrating the experinental ARPES over the polar angle and assuming azimuthal symmetry around the polarization axis. In absolute units, an increasing laser intensity results in the production of higher energy electrons. However, in energy units scaled to $U_{1}$, the PE distributions at ail intensities are similar in shape. If these three distributions are scaled such that their low energy maxima are made equal, one sees that the fraction in the high energy plateau drops with increasing intensity, scaling approximately as $I^{-2.5}$. As will be shown below, all these features form a consistent and qumntitatim picture of elastic rescattering of a 
field-driven electron in the tunneling limit.

Qualitatively, the PES in Figs. 1 and 2 provide some immediate insight into the significance of the spreading of the wave packet. As discussed earlier, classically approximately half the trajectories return to cross the plane of the nucleus. However, the transverse expansion causes most returning trajectories to have very large impact parameters, diluting the effect of rescattering. Our earlier numerical studies on neutral helium found that the returning wave packet at this wavelength has a radius of approximately $30 a_{0}$ as it rescatters from the nucleus. For a given atom this width does not depend on the laser intensity but only on the free propagation time and therefore only on the laser wavelength.

The quantitative comparisons of the measured and calculated total (spatial and temporal averaged) PE spectra are shown in Fig. 2. The dashed-dotted line in Fig. 2(b) is the calculated PES using the SQC model. The obvious failure of the model in predicting the high energy portion of the spectrum indicates the necessity of including rescattering. The dashed lines are the calculated PES using the $\mathrm{He}^{+}$potential and gives excellent agreement with the experimental measurement over the entire energy range for both "pure" tunneling cases. The poorer agreement seen in Fig. 2(c) signifies the transition into the mixed regime where the MPI contribution is becoming significant. Figure 2(b) also shows a PES calculated using a pure hydrogenic potential (dotted line). Coulomb scattering significantly underestimates the high energy plateau because the backscattering which produces the high energy electrons is most strongly affected by the more attractive short range part of the real potential.

These calculations also provide the angle-resolved PES. The calculated results are much smoother than the measured values, but reproduce the angle-dependent cutoffs in energy and the flatness of the high energy component. We obtain similarly good agreement for the other intensities studied except that at the highest intensity the measured PE spectrum ends around 10-20 percent lower energy than we predict. This may be due to poorer statistics in the measurement for the most energetic electrons at the highest intensity.

Angle-resolved PES results were also obtained for the ionization of neon by $0.78 \mu \mathrm{m}$ radiation. The ion and electron spectra show an evolution consistent with "pure" tunnel ionization near the saturation intensity. Results for both helium and neon are compiled in Fig. 3 along with calculated curves. The plot shows the ratio of electrons with energies $>2 U_{p}$ over those with energy $<2 U_{p}$ as a function of intensity. The open and filled stars are the ratio derived from the measured total PES for helium and neon, respectively. The three curves are the ratio obtained from the experimentally averaged calculations using Coulomb (dashed), $\mathrm{He}^{+}$(solid), and $\mathrm{Ne}^{+}$(dotted) potentials. The helium data agrees well with the calculated curve (solid line) for the two highest intensities but deviates away for the lowest intensity due to the multiphoton contributions which is not included in our calculation. The neon ratio point (filled star) shows that at the same intensity as helium, neon is approximately ten times more efficient at producing high energy electrons. This result is quite consistent with the observed ten fold increase in the high harmonic emission [15] for neon over helium. This difference is reflected in the calculated curves and again demonstrates the importance of the short range part of the potential in accurately reproducing the measured results at these intensities. Further evidence is seen for the Coulomb curve which underestimates the amount of elastic rescattering. However, as the intensity decreases the differences between all three curves vanish and 

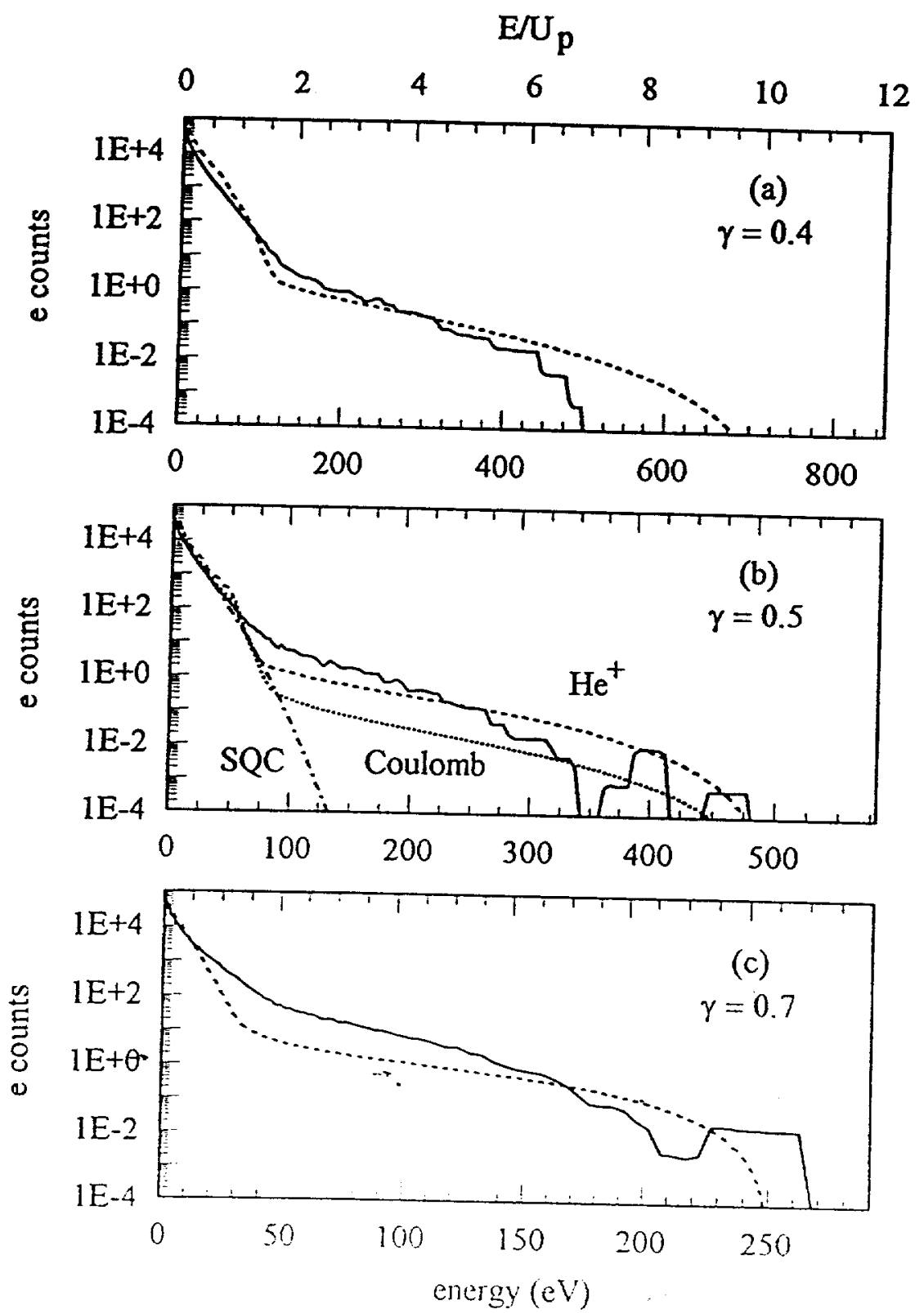

Figure 2. Total helium PE energy dist, ribution for $0.78 \mu \mathrm{m}$ excitation at (a) 12 , (b) 8 and (c) $4 \times 10^{14} \mathrm{~W} / \mathrm{cm}^{2}$. The experimental and calculated distributions using the complete semi-classical theory presented here correspond to the solid and dashed lines, respectively. In panel (b) the dotted line results from pure Coulomb rescattering and the dashed-dotted is without rescattering (SQC model). The Keldysh parameter; $i$, is indicated for each intensity. 


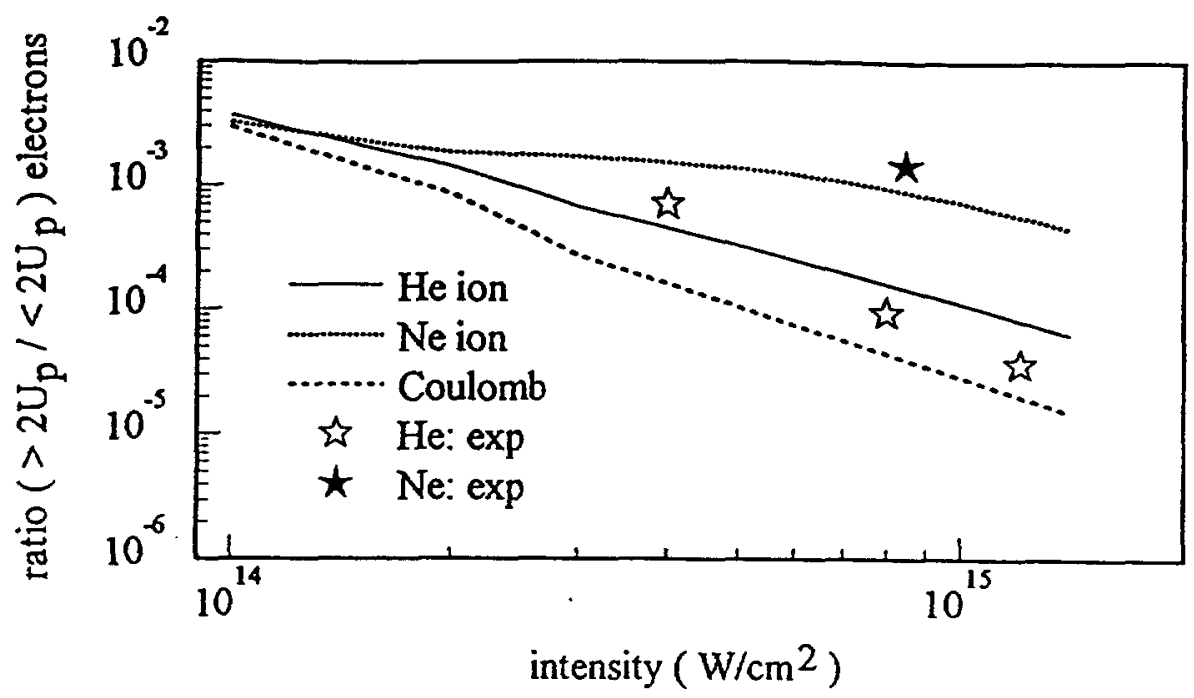

Figure 3. Compiled experimental and calculated ratio of total number of electrons with energies $>2 U_{p}$ over those $<2 U_{p}$.

in fact approach the Coulomb scattering result. Physically, decreasing intensity reduces the electron's return energy which equates to softer collisions, consequently diminishing the influence of the short range physics. Of course, experimental access to this regime is impossible due to the overwhelming contribution of MPI $(\gamma>1)$.

\section{Double ionization and the rescattering model}

Besides the collection of the photoelectrons, data was collected for the total ion yield as a function of intensity for both helium and neon. Walker et al [5] have demonstrated the versatility of kilohertz laser systems for increasing the sensitivity of total ion yield measurements. In fact, this measurement of the helium ion yield surpassed previous reports by five orders of magnitude. This data not only provides an accurate test of calculated rates but also offers some revealing insights into the physics of strong-field double or nonsequential (NS) ionization. For instance, the NS production of $\mathrm{He}^{2+}$ has been shown to be linked to the tumneling dynamics of the first electron, even when MPI dominates.

A sensitive measure of the nonsequential dynamics is provided by plotting the intensity dependence of the $\mathrm{X}^{2+} / \mathrm{X}^{+}$ratio $(\mathrm{X}=\mathrm{He}$ or $\mathrm{Ne}$ ) for both helium (solid circles) and neon (open circles) for $0.78 \mu m$ excitation, as shown in Fig. 4 . To ensure accuracy, the two ions are concurrently collected at a fixed intensity and averaged for at least $10^{6}$ laser shots. The plot shows that the measured NS yield is similar for both atoms, achieving a value of $0.0020[3]$ for helium and 0.0018 [4] for neon at their saturation intensity: Below saturation, the ratio of each decreases by approximately a factor of 10 over the measured intensity range, although the total rates are changing by seven orders of magnitude. Furthermore, analysis shows that both the helium and neon ratio scale with the tunneling fraction of the ionization, again providing an important clue to the NS dynamics. 


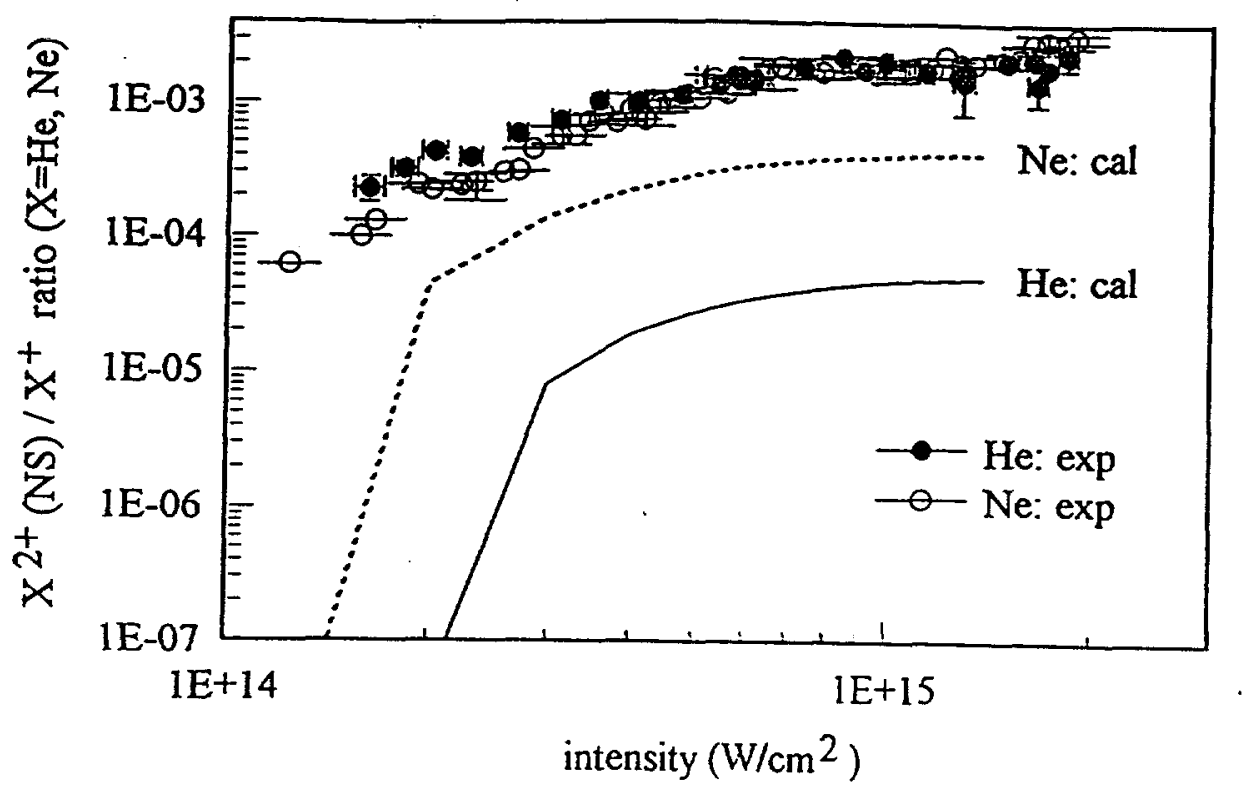

Figure 4. Compiled experimental and calculated ratio of nonsequential double ionization to single ionization for helium and neon for $0.78 \mu \mathrm{m}$ excitation.

The complete quasiclassical calculation, introduced in Section 2.2 , can be used to predict the double-to-single ionization ratio produced from e-2e inelastic rescattering. Figure 4 shows the results for both helium (solid line) and neon (dashed line). These results are calculated using the same initial conditions and core potentials used to calculate the photoelectron spectra of Figs. 2 and 3. A modified "field-free" e-2e Lotz cross-section [13] is used to account for double ionization contributions from both core excitation and direct ionization. In these strong fields, the returning electron needs only to excite one of the remaining electrons of the ion in order to produce the doubly charged ion. Any excited state will be immediately ionized when the oscillating field of the laser reaches its next maximum. Also, it has been shown [14] that the use of field-free cross sections is a reasonable approximation since the slowly varying electric field from the laser has a very small effect on the inelastic scattering processes. Clearly, the e-2e rescattering severely underestimates the absolute measured ratio, as well as the shape. The ratio of the experimental to calculated value at saturation is 47 for helium and 5 for neon. The lack of agreement is a clear indication that more than inelastic rescattering is involved in the physics of the nonsequential ionization. Reasonable assumptions on wave packet spreading and cross sections yield accurate predictions for the electron distributions seen in Fig. 2 and support the estimates used in our model.

The disagreement between the rescattering predictions and the experiment goes beyond underestimating the absolute value. As seen in Fig. 4, the experimental data shows a smooth decrease in the ratio with decreasing intensity, whereas the calculations show a sharp and abrupt cutoff. The origin of the cutoff is clear, as the intensity is lowered the electron's retum energy decreases to the point that it can no longer free the second electron. Again, no such behavior is seen in the experiment. Additionally, it is difficult to rationalize in a rescattering picture why the double ionization ratios would be the 


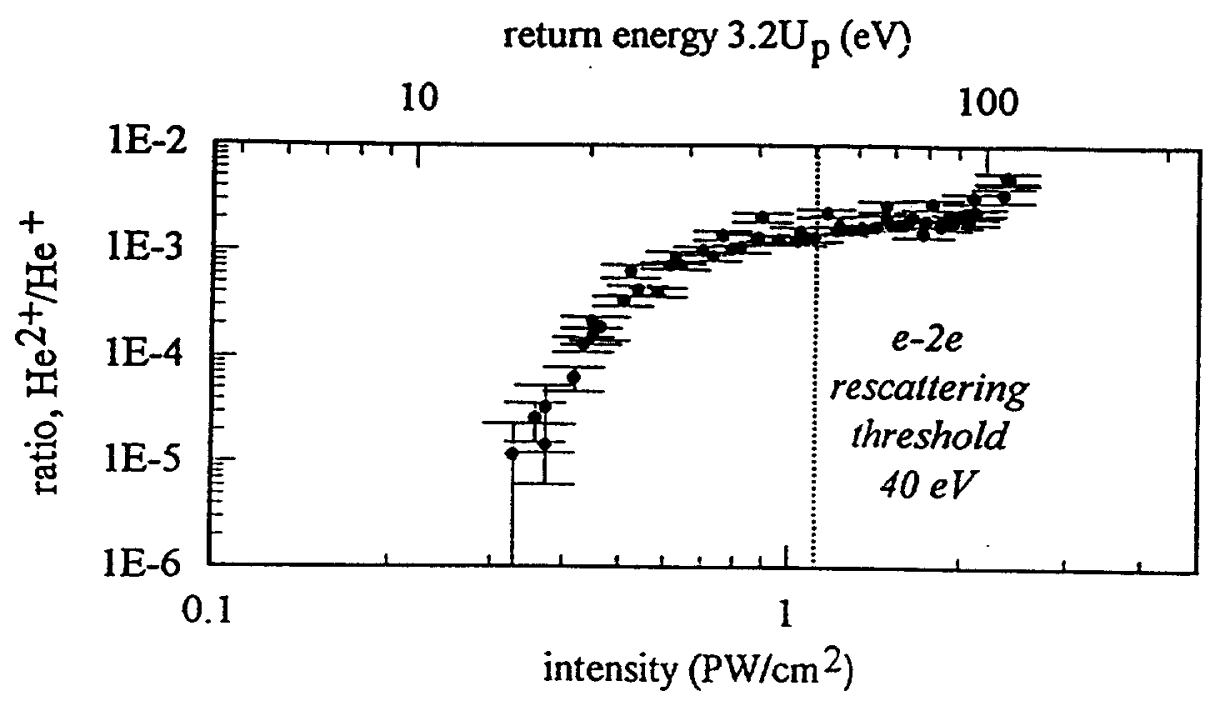

Figure 5. The ratio of double-to-single ionization for helium excited by a 0.39 $\mu m$ pulse. The upper abscissa scale gives the return energy at this wavelength and the dotted line marks the classical threshold.

same for helium and neon considering the difference in the e-2e cross sections. The good agreement found between the complete quasiclassical calculation and the experimental electron distributions demonstrated the important distinction produced by the atom's short-range potential. Obviously the calculated curves in Fig. 4 reflect the difference in the ionization cross sections, while the experiment does not.

A final piece of evidence that contradicts the predictions of the inelastic rescattering model for double ionization is shown in Fig. 5. The plot shows the ratio of $\mathrm{He}^{2+}(\mathrm{NS}) / \mathrm{He}^{+}$ as a function of intensity for $0.39 \mu \mathrm{m}$ excitation. The shorter wavelength reduces the ponderomotive energy by a factor of 4 , since it scales as $\omega^{-2}$, which consequently decreases the return energy. The dotted line shows the intensity below which the return energy can no longer excite the core electron, that is $3.2 U_{p}<40 \mathrm{eV}$, the excitation energy of the first excited state of helium. Clearly, all the nonsequential production is occurring below this threshold with a value $(0.0013)$ not much different from the $0.78 \mu \mathrm{m}$ result. Again these measurements reaffirm that inelastic rescattering camnot be the dominant mechanism for strong-field double ionization.

\section{Conclusion}

We have presented the first systematic study of the photoelectron distributions and double ionization in the strong-field tumneling regime. The distinctive electron energy and angular distributions are shown to be a clear signature of this limit. We showed that we could quantitatively reproduce these electron distributions using a complete quasiclassical model which includes the effects of a single recollision with the ion core. We show that to accurately reproduce the experimental results the details of the ion core's short range potential must be included in the rescattering calculations. Our analysis demonstrates that the strength of the ligh energy portion of the photoelectron spectrum is sensitive to the initial width of the tunneling wave packet. 'The agreement we 
have obtained supports the value we have determined and probably rules out anything substantially different from this.

In a consistent manner, calculation of the double ionization yield produced by $\mathrm{e}-2 \mathrm{e}$ inelastic rescattering severely underestimates the measured value for both helium and neon at $0.78 \mu \mathrm{m}$. Qualitatively, the measured ratio curves shows no resemblance to the threshold behavior or the core dependence expected from the rescattering picture. Furthermore, double ionization of helium persists at shorter wavelengths, $0.39 \mu \mathrm{m}$, at intensities well below the classical rescattering threshold for $\mathrm{e}-2 \mathrm{e}$ recollision.

Thus, all the evidence suggests that in the strong-field tunneling limit, the rescattering model captures, even quantitatively, the essential physics leading to the production of high energy electrons and harmonics. However, e-2e rescattering fails, evenly qualitatively, to reproduce most of the salient features of the experiment. We propose that strong-field double ionization is dominated by some other mechanism involving simultaneous two-electron ejection either through a shake-off [16] or threshold mechanism. We note that if the departure of the first electron is non-adiabatic in the sense that a second electron is left in an excited state orbital, this would appear as direct-double ionization. This is because, as we discussed above, the field is strong enough that it would immediately strip the excited electron when it reaches its next maximum. Unfortunately, the underlying mechanism for this process remains unclear, but various theoretical twoelectron $[17,18]$ treatments have begun. However, we emphasize that the results we have presented better defines the underlying dynamics of these processes and provides more stringent limits on any future models.

\section{Acknowledgments}

This research was carried out in part at Brookhaven National Laboratory under contract No. DE-AC02-76CH00016 with the U.S. Department of Energy and supported by its Division of Chemical Sciences, Office of Basic Energy Sciences, and in part under the auspices of the U. S. Department of Energy at the Lawrence Livermore National Laboratory under contract No. W-7405-ENG-48. L. F. D. and P. A. acknowledge travel support from NATO under Contract No. SA.5-2-05(RG910678).

\section{References}

[1] For a recent review, see DiMauro L F and Agostini P 1995 Advances in Atomic, Molecular, and Optical Physics 35 Bederson B and Walther H, Eds. (San Diego: Academic Press)

[2] Keldysh L V 1965 Sov. Phys. JETP 20 1307-1311

[3] Corkum P B, Burnett N H and Brunel F 1989 Plyys. Rev. Lett. 62 1259-1262

[4] Mohideen U, Sher M H, Tom H W K, Aumiller G D, Wood II O R, Freeman R R, Bokor J and Bucksbaum P H 1993 Phys. Rev. Lett. 71 509-512

[5] Walker B, Sheehy B, DiMauro L F, Agostini P, Schafer K J and Kulander K C 1994 Phys. Rev. Lett. 73 1227-1230

[6] Yang B, Schafer K J, Walker B, Kulander K C, Agostini P and DiMauro L F 1993 Phys. Rev. Lett. 71 3770-3773 
[7] Paulus G G, Becker W, Nicklich W and Walther H 1994 J. Phys. B: At. Mol. Phys. 27 L703-L708

[8] Schafer K J, Yang B, DiMauro L F and Kulander K C 1993 Phys. Rev. Lett. 70 1599-1602

[9] Corkum P B 1993 Phys. Rev. Lett. 71 1994-1997

[10] Lewenstein M, Kulander K C, Schafer K J and Bucksbaum P H 1995 Phys. Rev. A51 1495-1507

[11] Schiff L I 1968 Quantum Mechanics (New York: McGraw Hill)

[12] Ammosov M V, Delone N B and Krainov V P 1986 Sov. Phys. JETP 64 1191-1997

[13] Lotz W 1968 Z. Pliys. 216 241-247

[14] Kulander K C, Cooper J and Schafer K J 1995 Phys. Rev. A51 561-568

[15] L'Huillier A and Balcou P 1993 Phys. Rev. Lett. 70 774-777

[16] Fittinghoff D, Bolton P R, Chang B and Kulander K C 1992 Phys. Rev. Lett. 69 2642-2645

[17] Becker A and Faisal F H M 1996 J. Pliys. B: At. Mol. Phys. 29 L197-L202

[18] Watson J B, Sanpera A, Lappas D G, Knight P L and Burnett K 1996 Proceedings of ICOMP VII (Bristol: IOP) 


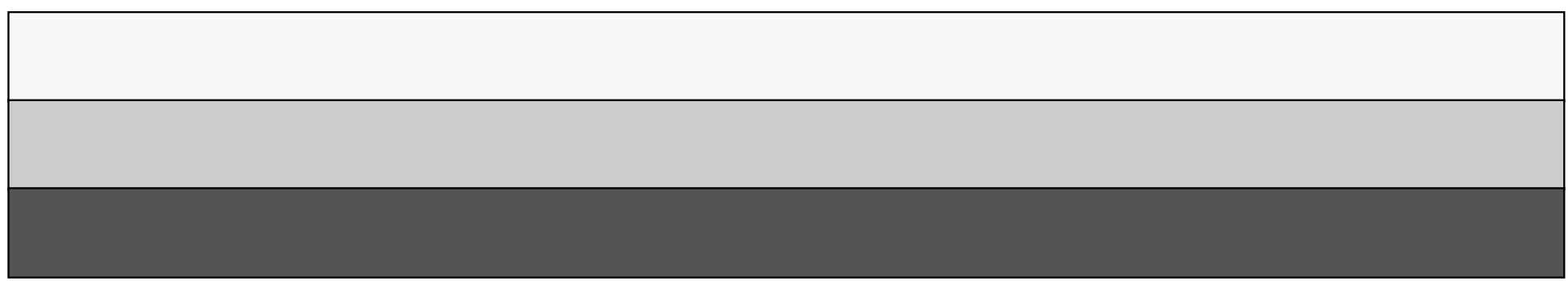

